\title{
Magnetic-free non-reciprocity and isolation based on parametrically modulated coupled-resonator loops
}

\author{
Nicholas A. Estep ${ }^{\dagger}$, Dimitrios L. Sounas ${ }^{\dagger}$, Jason Soric and Andrea Alù ${ }^{\star}$
}

Non-reciprocal components, which are essential to many modern communication systems, are almost exclusively based on magneto-optical materials, severely limiting their applicability. A practical and inexpensive route to magneticfree non-reciprocity could revolutionize radio-frequency and nanophotonic communication networks. Angular-momentum biasing was recently proposed as a means of realizing isolation for sound waves travelling in a rotating medium ${ }^{1}$, and envisaged as a path towards compact, linear integrated non-reciprocal electromagnetic components ${ }^{2,3}$. Inspired by this concept, here we demonstrate a subwavelength, linear radio-frequency non-reciprocal circulator free from magnetic materials and bias. The scheme is based on the parametric modulation of three identical, strongly and symmetrically coupled resonators. Their resonant frequencies are modulated by external signals with the same amplitude and a relative phase difference of $120^{\circ}$, imparting an effective electronic angular momentum to the system. We observe giant non-reciprocity, with up to six orders of magnitude difference in transmission for opposite directions. Furthermore, the device topology is tunable in real time, and can be directly embedded in a conventional integrated circuit.

Early attempts to realize magnetic-free non-reciprocity were based on the non-reciprocal properties of transistors at microwave frequencies ${ }^{4}$, and on networks of electro-optical modulators at optical frequencies ${ }^{5-8}$. However, such approaches traded the absence of magnetic bias with other significant drawbacks, such as the strong nonlinearities and poor noise-performance of transistors, or the large size and complexity of the required electro-optical networks. More recently, non-reciprocity has been achieved in transistor-loaded metamaterials ${ }^{9,10}$ and nonlinear devices ${ }^{11-14}$. Also these solutions impose severe restrictions on the input power levels, generally degrading the signal quality because of noise or signal distortion. Another interesting approach to magneticfree non-reciprocity has been introduced ${ }^{15}$, using asymmetric mode conversion in spatiotemporally modulated waveguides. This concept is especially attractive for integrated optical networks, as it may be fully realized in silicon photonics ${ }^{16}$. However, this technique and its variants ${ }^{17-22}$ lead to structures much larger than the wavelength, owing to the weak electro-optic or acousto-optic effects on which they rely, and require complex modulation schemes. In a similar context, the concept of a non-reciprocal device based on parametrically coupled resonators has been theoretically explored ${ }^{23}$.

An approach that can lead to compact, magnetic-free nonreciprocal devices with relaxed implementation complexity was recently introduced ${ }^{1-3}$, based on angular-momentum biasing of a resonant ring. Angular momentum can be applied either by mechanically spinning a fluid, as proved for acoustic waves ${ }^{1}$, or, more conveniently for electromagnetic waves, by spatiotemporal modulation with a travelling wave, realizing an effective electronic $\operatorname{spin}^{2,3}$, as illustrated in Fig. 1a. The resonant nature of the modulated ring can substantially boost the otherwise weak electrooptic effects through which spatiotemporal modulation is typically achieved $^{24,25}$, allowing the design of largely non-reciprocal devices with dimensions of the order of, or even smaller than the wavelength. Furthermore, in contrast to other approaches based on spatiotemporal modulation ${ }^{15,19}$, angular-momentum biasing is based on uniform modulation across the ring cross-section, thus significantly simplifying the fabrication process.

Inspired by these ideas, here we propose a device that provides strong magnetic-free non-reciprocity at the subwavelength scale

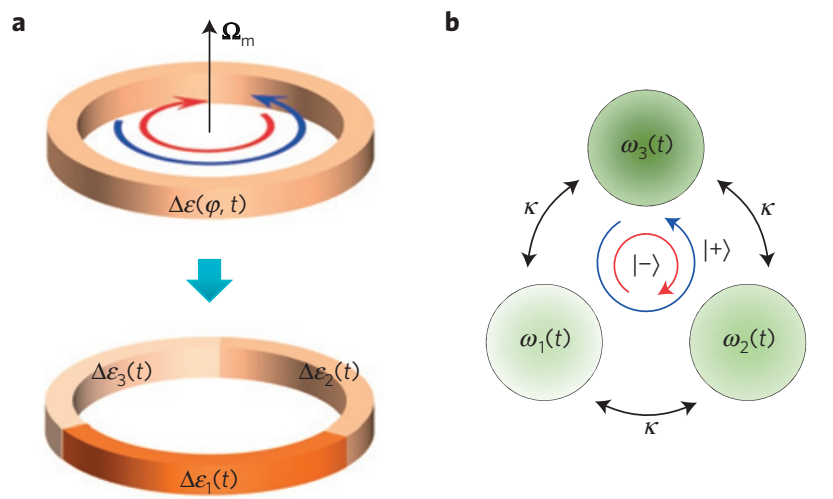

Figure 1 | Magnetic-free non-reciprocity with angular-momentum biasing. a, An azimuthally symmetric ring with spatiotemporal permittivity modulation $\Delta \varepsilon(\varphi, t)=\Delta \varepsilon_{\mathrm{m}} \cos \left(\omega_{\mathrm{m}} t-I_{\mathrm{m}} \varphi\right)$. The modulation imparts an effective electronic spin to the ring, with angular velocity $\Omega_{\mathrm{m}}=\omega_{\mathrm{m}} / \mathrm{I}_{\mathrm{m}}$, which lifts the degeneracy of counter-propagating resonant states and induces non-reciprocity. Its practical realization typically involves discretizing $\Delta \varepsilon$ into $N$ different regions (three in the figure). b, A loop consisting of three strongly coupled identical resonators, whose frequencies are modulated according to $\omega_{1}(t)=\omega_{0}+\delta \omega_{\mathrm{m}} \cos \left(\omega_{\mathrm{m}} t\right)$, $\omega_{2}(t)=\omega_{0}+\delta \omega_{\mathrm{m}} \cos \left(\omega_{\mathrm{m}} t+2 \pi / 3\right)$ and $\omega_{3}(t)=\omega_{0}+\delta \omega_{\mathrm{m}} \cos \left(\omega_{\mathrm{m}} t+4 \pi / 3\right)$. Modulation of the resonance frequencies lifts the degeneracy of the counter-rotating states $| \pm\rangle=\left[\begin{array}{lll}1 & \mathrm{e}^{ \pm i 2 \pi / 3} & \mathrm{e}^{ \pm i 4 \pi / 3}\end{array}\right]^{\top}$, producing nonreciprocity with $100 \%$ modulation efficiency. $\kappa$ is the coupling coefficient. 


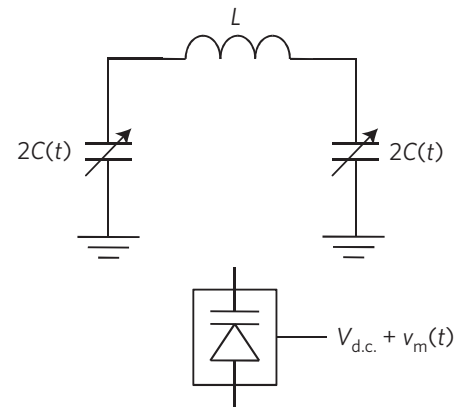

b

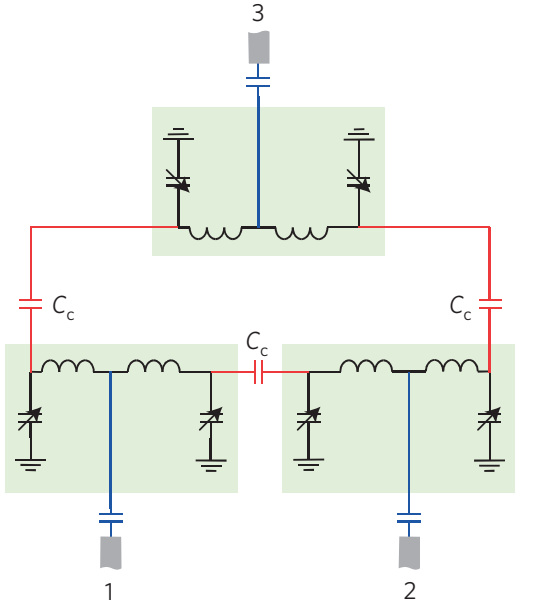

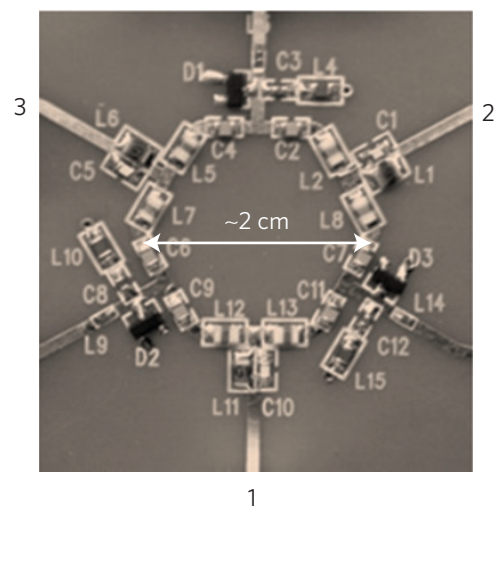

Figure $\mathbf{2}$ | Circuit implementation of the non-reciprocal coupled-resonator loop at radio frequencies. $\mathbf{a}$, A single resonant circuit of the proposed device: an $L-C$ tank with modulated capacitance. The capacitance is equally distributed at both sides of the inductance to maintain symmetry. Capacitance modulation is achieved with varactor diodes, controlled by a static signal $V_{\text {d.c. }}$ and the modulation signal $V_{m}(t)$. $\mathbf{b}, A$ loop formed by three identical resonators coupled through three identical capacitances $C_{c}$. The loop is further coupled to three external microstrip lines carrying the external signal. c, Fabricated prototype. The maximum dimension of the structure is $2 \mathrm{~cm}$, corresponding to an electrical size of $\lambda / 75$ at $200 \mathrm{MHz}$.

by dynamically modulating three identical resonant circuit tanks arranged in a loop and strongly coupled to each other, as in Fig. 1b. The resonant frequencies of the individual tanks are temporally modulated in a circularly rotating fashion as $\omega_{1}(t)=\omega_{0}+\delta \omega_{\mathrm{m}} \cos \left(\omega_{\mathrm{m}} t\right), \omega_{2}(t)=\omega_{0}+\delta \omega_{\mathrm{m}} \cos \left(\omega_{\mathrm{m}} t+2 \pi / 3\right)$ and $\omega_{3}(t)=\omega_{0}+\delta \omega_{\mathrm{m}} \cos \left(\omega_{\mathrm{m}} t+4 \pi / 3\right)$, where $\omega_{0}$ is the static value of the resonant frequency, $\delta \omega_{\mathrm{m}}$ is the modulation amplitude and $\omega_{\mathrm{m}}$ is the modulation frequency, so that an effective electronic spin is imparted to the system. Without modulation, the loop supports two degenerate counter-rotating modes, similar to the uniform ring of Fig. 1a. However, when the modulation is switched on, this degeneracy is lifted and non-reciprocity is induced. As we show in the following, this solution markedly boosts the modulation efficiency of the device compared to the case of a single modulated resonant ring, as considered in refs 2,3 , largely relaxing the requirements in terms of the modulation intensity and subsequently improving the overall efficiency of the structure.

In the absence of modulation $\left(\delta \omega_{\mathrm{m}}=0\right)$, the loop of Fig. $1 \mathrm{~b}$ supports three resonant states: a common state with state vector $|c\rangle=\left[\begin{array}{lll}1 & 1 & 1\end{array}\right]^{\mathrm{T}}$ and frequency $\omega_{c}=\omega_{0}+2 \kappa$, and two degenerate right- and left-handed states with state vectors $| \pm\rangle=\left[\begin{array}{lll}1 & \mathrm{e}^{ \pm i 2 \pi / 3} & \mathrm{e}^{ \pm i 4 \pi / 3}\end{array}\right]^{\mathrm{T}}$ and frequencies $\omega_{ \pm}=\omega_{0}-\kappa$, where $\kappa$ is the coupling coefficient. The applied modulation mixes rightand left-handed states, producing two new hybrid states

$$
\begin{aligned}
& |R\rangle=|+\rangle \mathrm{e}^{-i \omega_{\mathrm{R}} t}-\frac{\Delta \omega}{\delta \omega_{\mathrm{m}}}|-\rangle \mathrm{e}^{-i\left(\omega_{R}-\omega_{\mathrm{m}}\right) t} \\
& |L\rangle=|-\rangle \mathrm{e}^{-i \omega_{L} t}+\frac{\Delta \omega}{\delta \omega_{\mathrm{m}}}|+\rangle \mathrm{e}^{-i\left(\omega_{L}+\omega_{\mathrm{m}}\right) t}
\end{aligned}
$$

where $\quad \Delta \omega=\sqrt{\omega_{\mathrm{m}}^{2}+\delta \omega_{\mathrm{m}}^{2}}-\omega_{\mathrm{m}}, \quad \omega_{R}=\omega_{ \pm}-\Delta \omega / 2$ and $\omega_{L}=\omega_{ \pm}+\Delta \omega / 2$, as analytically derived in the Methods for $\omega_{\mathrm{m}} \ll \omega_{\mathrm{c}}-\omega_{ \pm}$.

It is interesting to observe that both $|R\rangle$ and $|L\rangle$ consist of a dominant state at $\omega_{R, L}$ and a secondary state, red- or blueshifted by $\omega_{\mathrm{m}}$, as in the case of the uniformly modulated ring of refs 2,3 . However, despite this apparent resemblance in the form of $|R\rangle$ and $|L\rangle$, the mechanism that creates the frequency separation $\Delta \omega$ between resonant states in the geometry analysed here is significantly different from the uniformly modulated ring of refs 2,3. For a uniform resonant ring (Fig. 1a), $\Delta \omega$ is the result of an azimuthally travelling wave modulation $\Delta \varepsilon_{\mathrm{m}} \cos \left(\omega_{\mathrm{m}} t-l_{\mathrm{m}} \varphi\right)$, where $\Delta \varepsilon_{\mathrm{m}}$ and $l_{\mathrm{m}}$ are the modulation amplitude and azimuthal order, respectively. If $l_{\mathrm{m}}=2 l$, where $l$ is the resonant order of the ring, $\delta \omega_{\mathrm{m}}=\omega_{l} \Delta \varepsilon_{\mathrm{m}} /(2 \varepsilon)$ (refs 2,3), where $\omega_{l}$ is the resonant frequency and $\varepsilon$ the static permittivity. The ideal continuous modulation assumed in Fig. 1a (top) is difficult, if not impossible, to realize, and an angular discretization is typically required. A discrete modulation profile with $N$ different modulation regions, as sketched in Fig. 1a (bottom), is equivalent to a continuous effective modulation with reduced effective amplitude $\Delta \varepsilon_{\mathrm{m} \text {,eff }}=\Delta \varepsilon_{\mathrm{m}} \operatorname{sinc}(2 l / N)$ (ref. 3), revealing a fundamental trade-off between fabrication complexity, proportional to $N$, and non-reciprocal response. As an example, for $N=3$-that is, the minimum value for which $\Delta \varepsilon_{\mathrm{m}, \text { eff }} \neq 0$ - and $l=50-$ a typical value in realistic high- $Q$ micro-ring resonators at $1.55 \mu \mathrm{m}-\Delta \varepsilon_{\mathrm{m}, \text { fff }}=0.008 \Delta \varepsilon_{\mathrm{m}}$, implying that only $0.8 \%$ of the modulation signal is effectively used to generate non-reciprocity.

In contrast, in the composite resonant loop of Fig. $1 \mathrm{~b}$ the frequency splitting is achieved by modulating the frequency of each resonator, which can be obtained by applying a uniform permittivity modulation with amplitude $\Delta \varepsilon_{\mathrm{m}}$ all across each resonator ${ }^{24}$, leading to $\delta \omega_{\mathrm{m}}=\omega_{0} \Delta \varepsilon_{\mathrm{m}} /(2 \varepsilon)$, as shown in the Methods. Remarkably, in this topology the entire modulation signal is used to produce the frequency separation $\Delta \omega$, indicating that maximum (unitary) modulation efficiency is achieved in a simple fabrication scheme requiring only three independent modulation regions.

We realized the structure of Fig. $1 \mathrm{~b}$ at $\mathrm{RF}$ using three basic $L-C$ circuit tanks, as in Fig. 2a, where the capacitance $C$ is equally distributed on both sides of the inductance $L$ to maintain a symmetric structure (see Supplementary Methods for a full schematic of the realized circuit). The resonance frequency modulation is achieved by means of capacitance modulation, commonly obtained in RF with varactor diodes. These diodes are biased by two signals, a static signal $V_{\text {d.c. }}$, which provides the required reverse bias and controls the static capacitance, and a RF signal $v_{\mathrm{m}}$ with frequency $\omega_{\mathrm{m}}$ and amplitude $V_{\mathrm{m}}$, providing the time modulation. Assuming that the resonators are coupled to each other through capacitances $C_{c}$, as in Fig. $2 \mathrm{~b}$, the frequencies of the common and rotating states are $\omega_{\mathrm{c}}=\omega_{0} / \sqrt{1+2 C_{\mathrm{c}} / C}$ and $\omega_{ \pm}=\omega_{0} \sqrt{\left(C+3 C_{c} / 2\right) /\left(C+2 C_{c}\right)}$, respectively, where $\omega_{0}=1 / \sqrt{L C}$ is the static resonance frequency of each tank. Then, if the amplitude of the capacitance modulation is $\Delta C_{\mathrm{m}}$, the frequency modulation 

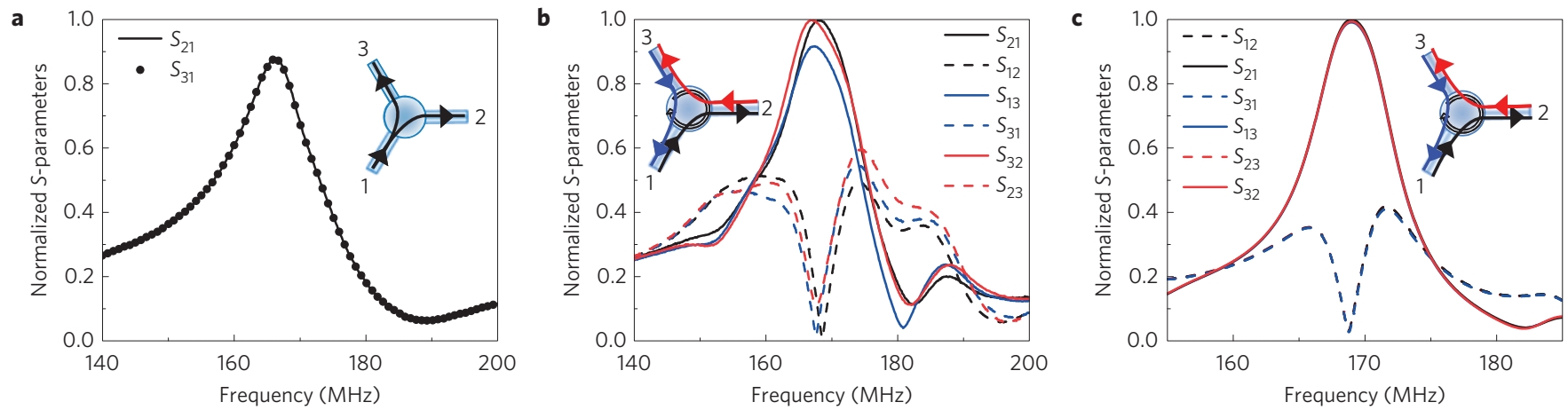

Figure 3 | Response versus frequency. a, Measured transmission from port 1 to ports 2 and 3 without modulation ( $V_{m}=0 \mathrm{~V}$ ). The power is equally split to the output ports. b. Measured scattering parameters when $V_{\mathrm{m}}=0.6 \mathrm{~V}$. Incident power to ports 1, 2 and 3 is transmitted to ports 2,3 and 1 , respectively, thus realizing a three-port circulator. c, Simulated response of the loop for the case of $\mathbf{b}$ : excellent agreement between theory and experiment is observed. All results correspond to $V_{\text {d.c. }}=1.99 \mathrm{~V}$

amplitude is found as $\delta \omega_{\mathrm{m}}=\omega_{ \pm} \Delta C_{\mathrm{m}} /(2 C)$. The frequency $\omega_{\mathrm{c}}$ should be designed to be as far as possible from $\omega_{ \pm}$in order for the common mode not to affect the operation of the structure at $\omega_{ \pm}$, at which non-reciprocity occurs. In the lumped-element circuit of Fig. 2b this condition is satisfied by taking $C_{c} \rightarrow \infty$, or equivalently by coupling the tanks through short circuits, yielding $\omega_{c}=0$ and $\omega_{ \pm}=\omega_{0} \sqrt{3} / 2$.

The non-reciprocal response of the circuit of Fig. $2 b$ is demonstrated by capacitively coupling it to three microstrip transmission lines, realizing a three-port device. Exciting the structure from, for example, port 1 at frequency $\omega_{ \pm}$results in the excitation of $|R\rangle$ and $|L\rangle$ with the same amplitude and opposite phase $\phi_{R}=-\phi_{L}$, owing to the symmetrical distribution of these states around $\omega_{ \pm}$. Then, the signals at ports 2 and 3 are proportional to $\mathrm{e}^{i 2 \pi / 3} \mathrm{e}^{i \phi_{R}}+\mathrm{e}^{i 4 \pi / 3} \mathrm{e}^{i \phi_{L}}$ and $\mathrm{e}^{-i 2 \pi / 3} \mathrm{e}^{i \phi_{R}}+\mathrm{e}^{-i 4 \pi / 3} \mathrm{e}^{i \phi_{L}}$, respectively, as the superposition of $|R\rangle$ and $|L\rangle$ at these ports. If $\delta \omega_{\mathrm{m}}$ and $\omega_{\mathrm{m}}$ are selected so that $\phi_{R}=-\phi_{L}=\pi / 6$, the signal at port 3 is identically zero, while the signal at port 2 is non-zero, routing the incident power from port 1 to port 2. Owing to the symmetry of the structure with respect to its ports, incident power from ports 2 and 3 is similarly routed to ports 3 and 1, thus realizing the functionality of a non-reciprocal circulator with infinite isolation. Notice that the above description assumes a weak excitation of the common state, which makes clear the importance of choosing its resonance frequency as far as possible from the resonance frequency of the rotating states.

The realized device was designed to resonate at $170 \mathrm{MHz}$ with a $Q$-factor of about 10 for $V_{\text {d.c. }}=1.99 \mathrm{~V}$ and $V_{\mathrm{m}}=0$. The modulation frequency was set to $15 \mathrm{MHz}$, in order for the intermodulation by-products at frequencies $\omega \pm \omega_{\mathrm{m}}$, created by the secondary substates of $|R\rangle$ and $|L\rangle$, to fall outside the resonance band, whose bandwidth is here around $10 \mathrm{MHz}$. The exact values of the circuit components and its full topology are provided in the Methods and Supplementary Methods. Figure $2 \mathrm{c}$ shows a photograph of the fabricated prototype. We underline here the deeply subwavelength size of the realized device $(\sim \lambda / 75)$, simply based on three lumped resonant circuit tanks.

Without modulation, the signal is equally split at the two output ports, as expected from symmetry, and the system is fully reciprocal (Fig. 3a). When the modulation signal is switched on, the symmetry is broken and power is unequally split. By varying the modulation amplitude it is possible to find a value for which the signal entering port 1 is routed exclusively to port 2 , corresponding to $\phi_{R}=-\phi_{L}=\pi / 6$. This condition is satisfied for $V_{\mathrm{m}}=0.6 \mathrm{~V}$, as can be seen in Fig. 3b: at the resonance frequency of $170 \mathrm{MHz}$, power incident to ports 1, 2 and 3 is routed to ports 2, 3 and 1, respectively, demonstrating the operation of an ideal, magnetic-free, deeply subwavelength linear circulator. For comparison, Fig. $3 c$ shows the
$S$-parameters obtained using full-wave and circuit simulations: the agreement with the measurement is excellent.

To get a deeper insight into the effect of $V_{\mathrm{m}}$ on the device operation, Fig. $4 \mathrm{a}$ shows the transmission between ports 1 and 2 at resonance versus $V_{\mathrm{m}}$. For $V_{\mathrm{m}}=0, S_{21}=S_{12}$, as expected. Increasing $V_{\mathrm{m}}$ results in an increase of $S_{21}$ and a decrease of $S_{12}$ until $V_{\mathrm{m}}=0.6 \mathrm{~V}$, where $S_{12}=0$. Past this point, $S_{21}$ and $S_{12}$ get closer, as expected when we depart from the destructive interference condition. For very large values of $V_{\mathrm{m}}, S_{21}$ and $S_{12}$ both tend to zero, because the counter-rotating states move far from $\omega_{ \pm}$and, therefore, are weakly excited at $\omega_{ \pm}$. The magnitude of the asymmetry between $S_{21}$ and $S_{12}$ is measured by the isolation $\left|S_{21} / S_{12}\right|$, plotted in Fig. $4 \mathrm{~b}$ on a logarithmic scale versus $V_{\mathrm{m}}$. At the optimum modulation voltage $V_{\mathrm{m}}=0.6 \mathrm{~V}, S_{21}$ is over four orders of magnitude larger than $S_{12}$, indicating giant non-reciprocity, well above the levels of any commercial magnetic-based device.

Another unique property of the proposed device consists in its real-time tunability features. The biasing voltage $V_{\text {d.c. }}$, which provides the reverse biasing condition for the varactor diodes, determines their static capacitance. Therefore, $V_{\text {d.c. }}$ can be used to actively control the static resonance frequency of the $L-C$ tanks, and consequently the frequency band over which non-reciprocity occurs. Figure 4c shows the measured isolation versus frequency for $V_{\text {d.c. }}$ varied between $1.73 \mathrm{~V}$ and $4.5 \mathrm{~V}$. The non-reciprocal response of our device can be efficiently tuned between $150 \mathrm{MHz}$ and $210 \mathrm{MHz}$, corresponding to a relative bandwidth of over $30 \%$. Across all this range, our measured isolation is above $40 \mathrm{~dB}$. This strong tuning capability is an additional advantage of the proposed device compared to conventional magnetic-based microwave circulators, and it may be exploited in scenarios requiring dynamic tuning to balance changes in temperature or in the environment. The electronic spin applied to the proposed coupled-resonator loop realizes the equivalent of a dynamically tunable, strongly biased ferromagnetic metamaterial substrate.

In addition to being an ideal replacement for microwave nonreciprocal components, with significant advantages in terms of size, integration, cost, linearity, tunability and noise reduction, our findings may become even more important when applied to different frequencies or other types of waves, such as light or sound. For instance, this concept may be disruptive for integrated nanophotonic technology, for which optical non-reciprocal components are critical for laser protection and signal routing. At visible frequencies, electro-optic modulation in silicon-based components is typically achieved by means of carrier injection/depletion ${ }^{24,25}$, which can provide relatively strong permittivity modulation, but is typically accompanied by significant loss and low modulation frequencies for large modulation amplitudes. These side effects impose limitations 

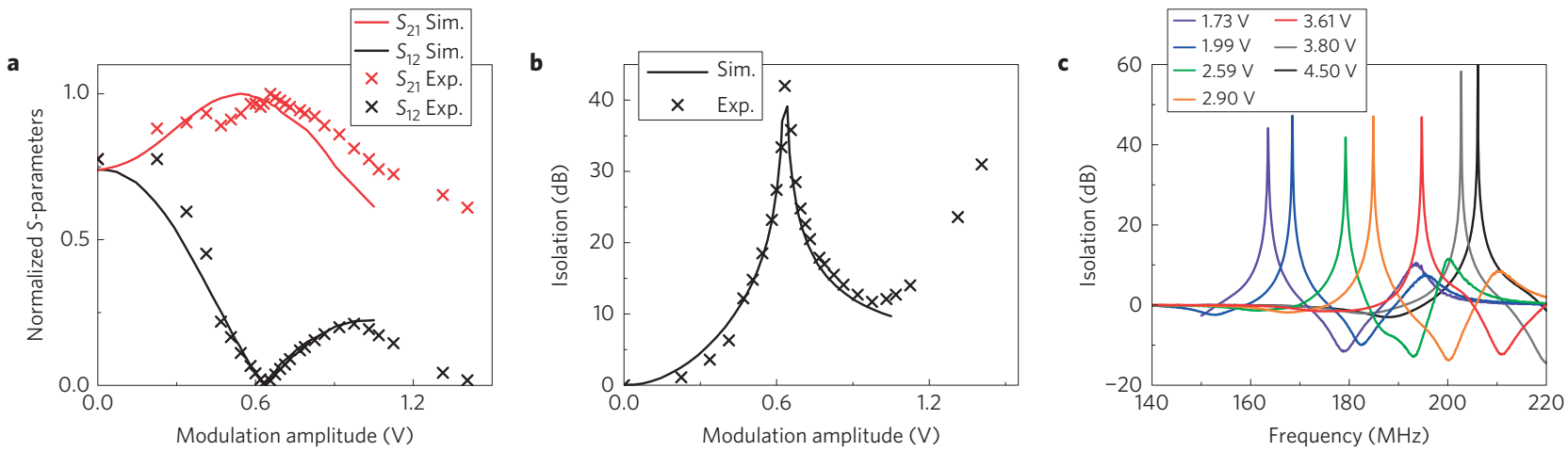

Figure 4 | Response versus modulation and static voltages. a, Measured and simulated transmission between ports 1 and 2 . Transmission is different for opposite propagation directions, indicating non-reciprocity. Maximum contrast is observed for $V_{\mathrm{m}}=0.6 \mathrm{~V}$. The results correspond to $V_{\text {d.c. }}=1.99 \mathrm{~V}$. The differences between the measured and simulated results are not due to experimental uncertainties, which are negligible here, but due to the tolerances in the values of the lumped elements which make the measured and simulated layouts slightly different. $\mathbf{b}$, Isolation $\left(S_{21} / S_{12}\right)$ in logarithmic scale for the same biasing conditions as in a. For $V_{m}=0.6 \mathrm{~V}$, the difference between $S_{12}$ and $S_{21}$ is over four orders of magnitude. $\mathbf{c}$, Measured isolation on a logarithmic scale versus frequency for different values of $V_{d . c}$. In all cases, $V_{m}$ is selected, based on our theory, so that isolation at resonance is maximum.

on the applicability of the principle of angular-momentum biasing in uniform micro-ring resonators ${ }^{2,3}$, as discussed before. In contrast, the concept presented here ensures maximum modulation efficiency, significantly relaxing the requirements in terms of modulation amplitude. This in turn allows large quality factors and large modulation frequencies, which translate into strong non-reciprocal response in deeply subwavelength devices. We also envisage the realization of the proposed rotating modulation of coupled-resonator loops in photonic crystal technology, for which high-Q-factor coupled cavities may be implemented and efficiently modulated ${ }^{26}$. Our study also represents a new demonstration of the exciting possibilities offered by dynamic modulation of coupled-resonator networks, with unique control over the flow of light, in the context of recently presented concepts of photonic topological edge states and effective magnetic fields for photons ${ }^{27-29}$.

\section{Methods}

Modes of the coupled-resonators loop. The coupled-mode equations of the system of Fig. 1 read

$$
\begin{aligned}
& \dot{a}_{1}=-i \omega_{1} a_{1}-i \kappa a_{2}-i \kappa a_{3} \\
& \dot{a}_{2}=-i \omega_{2} a_{2}-i \kappa a_{1}-i \kappa a_{3} \\
& \dot{a}_{3}=-i \omega_{3} a_{3}-i \kappa a_{1}-i \kappa a_{2}
\end{aligned}
$$

where $a_{1}, a_{2}$ and $a_{3}$ are the complex amplitudes of the three resonators. Equation (2) can be written in the more compact form

$$
\frac{\mathrm{d}}{\mathrm{d} t}|\psi\rangle=-i \Omega|\psi\rangle
$$

where $|\psi\rangle=\left[\begin{array}{lll}a_{1} & a_{2} & a_{3}\end{array}\right]^{\mathrm{T}}$ is the state vector of the system and

$$
\Omega=\left[\begin{array}{ccc}
\omega_{1} & \kappa & \kappa \\
\kappa & \omega_{2} & \kappa \\
\kappa & \kappa & \omega_{3}
\end{array}\right]
$$

In the absence of modulation, $\omega_{1}, \omega_{2}$ and $\omega_{3}$ are equal to $\omega_{0}$. Then, the eigenfrequencies and the corresponding state vectors of the system are found by the eigenvalues and eigenvectors of $\Omega$, respectively. In particular, it can be shown that there are three states, a common state with resonant frequency $\omega_{\mathrm{c}}=\omega_{0}+2 \kappa$ and state vector $|c\rangle=(1 / \sqrt{3})\left[\begin{array}{ccc}1 & 1 & 1\end{array}\right]^{\mathrm{T}}$, a right-handed rotating state with resonant frequency $\omega_{+}=\omega_{0}-\kappa$ and state vector $|+\rangle=(1 / \sqrt{3})\left[\begin{array}{lll}1 & e^{i 2 \pi / 3} & e^{i 4 \pi / 3}\end{array}\right]^{\mathrm{T}}$, and a left-handed rotating state with resonant frequency $\omega_{-}=\omega_{+}$and state vector $|-\rangle=(1 / \sqrt{3})\left[\begin{array}{lll}1 & \mathrm{e}^{i 4 \pi / 3} & \mathrm{e}^{i 2 \pi / 3}\end{array}\right]^{\mathrm{T}}$. Note that because the eigenvalues $\omega_{+}$and $\omega_{-}$are degenerate, the eigenvectors $|+\rangle$and $|-\rangle$ are not the only ones corresponding to these eigenvalues. As a matter of fact, any linear combination of $|+\rangle$ and $|-\rangle$, such as the vectors $\left[\begin{array}{lll}1 & -1 & 0\end{array}\right]^{\mathrm{T}}$ and $\left[\begin{array}{lll}1 & 0 & -1\end{array}\right]^{\mathrm{T}}$, are also valid eigenvectors of $\omega_{+}$and $\omega_{-}$. However, hereafter, we will use $|+\rangle$ and $|-\rangle$, because they bear an immediate physical meaning as counter-rotating states of the coupled-resonators loop, and they significantly simplify the mathematical analysis.

In the presence of modulation $\omega_{1}(t)=\omega_{0}+\delta \omega_{\mathrm{m}} \cos \left(\omega_{\mathrm{m}} t\right)$, $\omega_{2}(t)=\omega_{0}+\delta \omega_{\mathrm{m}} \cos \left(\omega_{\mathrm{m}} t+2 \pi / 3\right)$ and $\omega_{3}(t)=\omega_{0}+\delta \omega_{\mathrm{m}} \cos \left(\omega_{\mathrm{m}} t+4 \pi / 3\right)$ result in a time-dependent $\Omega$. In this case, the eigenstates of the system cannot be found by the eigenvalues and eigenvectors of $\Omega$, and a full solution of equation (3) is necessary. Because modulation constitutes a perturbation of the coupled system, it is convenient to express the eigenstates of the modulated system in terms of the eigenstates of the coupled non-modulated system. To this end, equation (3) is expressed in the basis of $|c\rangle,|+\rangle$ and $|-\rangle$, by multiplying $|\psi\rangle$ with the conjugate transpose of the matrix

$$
U=\frac{1}{\sqrt{3}}\left[\begin{array}{ccc}
1 & 1 & 1 \\
1 & \mathrm{e}^{i 2 \pi / 3} & \mathrm{e}^{i 4 \pi / 3} \\
1 & \mathrm{e}^{i 4 \pi / 3} & \mathrm{e}^{i 2 \pi / 3}
\end{array}\right]
$$

with columns the state vectors $|c\rangle,|+\rangle$ and $|-\rangle$. In the new basis, $|\psi\rangle=\left[\begin{array}{lll}a_{\mathrm{c}} & a_{+} & a_{-}\end{array}\right]^{\mathrm{T}}$, where $a_{\mathrm{c}}, a_{+}$and $a_{-}$are the complex amplitudes of the common, right- and left-handed states, respectively, and

$$
\Omega=\Omega_{0}+\delta \Omega
$$

where

$$
\Omega_{0}=\left[\begin{array}{ccc}
\omega_{\mathrm{c}} & 0 & 0 \\
0 & \omega_{+} & 0 \\
0 & 0 & \omega_{-}
\end{array}\right] \quad \delta \Omega=\frac{\delta \omega_{\mathrm{m}}}{2}\left[\begin{array}{ccc}
0 & \mathrm{e}^{-i \omega_{\mathrm{m}} t} & \mathrm{e}^{i \omega_{\mathrm{m}} t} \\
\mathrm{e}^{i \omega_{\mathrm{m}} t} & 0 & \mathrm{e}^{-i \omega_{\mathrm{m}} t} \\
\mathrm{e}^{-i \omega_{\mathrm{m}} t} & \mathrm{e}^{i \omega_{\mathrm{m}} t} & 0
\end{array}\right]
$$

If $\omega_{\mathrm{m}} \ll\left|\omega_{\mathrm{c}}-\omega_{ \pm}\right|$, coupling between the common and rotating states, corresponding to the first row and column of $\delta \Omega$, can be neglected and equation (3) simplifies to

$$
\begin{aligned}
& i \ddot{a}_{+}=\omega_{+} a_{+}+\frac{\delta \omega_{\mathrm{m}}}{2} \mathrm{e}^{-i \omega_{\mathrm{m}} t} a_{-} \\
& i \ddot{a}_{-}=\omega_{-} a_{-}+\frac{\delta \omega_{\mathrm{m}}}{2} \mathrm{e}^{i \omega_{\mathrm{m}} t} a_{+}
\end{aligned}
$$

It can be seen that equation (4) is satisfied if

$$
\begin{aligned}
& a_{+}=A_{+} \mathrm{e}^{-i \omega t} \\
& a_{-}=A_{-} \mathrm{e}^{-i\left(\omega-\omega_{\mathrm{m}}\right) t}
\end{aligned}
$$

where $A_{+}, A_{-}$and $\omega$ are constants. Then, equation (4) becomes

$$
\left[\begin{array}{cc}
\omega_{+} & \delta \omega_{\mathrm{m}} / 2 \\
\delta \omega_{\mathrm{m}} / 2 & \omega_{-}+\omega_{\mathrm{m}}
\end{array}\right]\left[\begin{array}{l}
A_{+} \\
A_{-}
\end{array}\right]=\omega\left[\begin{array}{l}
A_{+} \\
A_{-}
\end{array}\right]
$$


which is an eigenvalue problem with respect to $\omega$. The eigensolutions of this problem yield the eigenstates of the modulated system, as given by equation (1).

Modulation amplitude of the coupled-resonators system. We assume a resonator whose modal distribution fully resides in materials with permittivity $\varepsilon$, permeability $\mu$, and resonant frequency $\omega_{0}$. It can be shown from perturbation theory that a small change $\Delta \varepsilon$ of the permittivity results in the following change of the resonance frequency ${ }^{30}$

$$
\omega-\omega_{0} \approx-\omega_{0} \frac{\int \Delta \varepsilon\left|\mathbf{E}_{0}\right|^{2} \mathrm{~d} V}{\int\left(\varepsilon\left|\mathbf{E}_{0}\right|^{2}+\mu\left|\mathbf{H}_{0}\right|^{2}\right) \mathrm{d} V}
$$

where $\mathbf{E}_{0}$ and $\mathbf{H}_{0}$ are the resonant electric and magnetic field, respectively, and integration is performed all over the volume of the resonator. Furthermore, it is known that at resonance the electric and magnetic energies are equal, hence $\int \varepsilon\left|\mathbf{E}_{0}\right|^{2} \mathrm{~d} V=\int \mu\left|\mathbf{H}_{0}\right|^{2} \mathrm{~d} V$ and

$$
\omega-\omega_{0} \approx-\omega_{0} \frac{\int \Delta \varepsilon\left|\mathbf{E}_{0}\right|^{2} \mathrm{~d} V}{2 \int \varepsilon\left|\mathbf{E}_{0}\right|^{2} \mathrm{~d} V}
$$

If, in addition, $\varepsilon$ and $\Delta \varepsilon$ are uniform over the volume where most of the resonator's energy is concentrated, $\int \Delta \varepsilon\left|\mathbf{E}_{0}\right|^{2} \mathrm{~d} V \approx \Delta \varepsilon \int\left|\mathbf{E}_{0}\right|^{2} \mathrm{~d} V$ and $\int \varepsilon\left|\mathbf{E}_{0}\right|^{2} \mathrm{~d} V \approx \varepsilon \int\left|\mathbf{E}_{0}\right|^{2} \mathrm{~d} V$, showing that the frequency modulation, $\delta \omega_{\mathrm{m}}$, for a permittivity modulation amplitude $\Delta \varepsilon_{\mathrm{m}}$ is equal to $\delta \omega_{\mathrm{m}}=\omega_{0} \Delta \varepsilon /(2 \varepsilon)$.

Description of the experimental set-up. The complete experimental set-up is shown in Supplementary Fig. 2 and a list of the associated equipment is provided in Supplementary Table 2. A waveform generator provides the modulation signal, which is split into three equal parts by means of a power divider. The output signals are then led to three phase shifters, which provide the required phase difference of $120^{\circ}$ for the modulation signals of the three coupled resonators. The phase shifters are powered by a d.c. source and their phase shift is controlled by potentiometers. The outputs of the phase shifters are connected to the low-pass ports of three diplexers, whose outputs are connected to the RF/modulation ports of the ring. The high-pass ports of two of the diplexers are connected to the VNA ports while the high-pass port of the third diplexer is terminated to a matched load. The diplexers combine the modulation and RF signals and at the same time provide infinite isolation between the RF and modulation paths. By rotating the diplexers, which are connected to the VNA ports, it is possible to measure all the $S$-parameters of the circuit. The d.c. signal for biasing of the varactors is provided by a d.c. source connected to ports 4,5 and 6 of the ring.

Received 26 May 2014; accepted 17 September 2014; published online 10 November 2014

\section{References}

1. Fleury, R., Sounas, D. L., Sieck, C. F., Haberman, M. R. \& Alù, A. Sound isolation and giant linear nonreciprocity in a compact acoustic circulator. Science 343, 516-519 (2014).

2. Sounas, D. L., Caloz, C. \& Alù, A. Giant non-reciprocity at the subwavelength scale using angular momentum-biased metamaterials. Nature Commun. 4, 2407 (2013).

3. Sounas, D. L. \& Alù, A. Angular-momentum-biased nanorings to realize magnetic-free integrated optical isolation. ACS Photon. 1, 198-204 (2014).

4. Tanaka, S., Shimimura, N. \& Ohtake, K. Active circulators-The realization of circulators using transistors. Proc. IEEE 53, 260-267 (1965).

5. Hwang, I. K., Yun, S. H. \& Kim, B. Y. All-fiber-optic nonreciprocal modulator. Opt. Lett. 22, 507-509 (1997).

6. Bhandare, S. et al. Novel nonmagnetic 30-dB traveling-wave single-sideband optical isolator integrated in III/V material. IEEE J. Sel. Top. Quantum Electron. 11, 417-421 (2005)

7. Galland, C., Ding, R., Harris, N. C., Baehr-Jones, T. \& Hochberg, M. Broadband on-chip optical non-reciprocity using phase modulators. Opt. Express 21, 14500-14511 (2013).

8. Doerr, C. R., Chen, L. \& Vermeulen, D. Silicon photonics broadband modulation-based isolator. Opt. Express 22, 4493-4498 (2014).
9. Kodera, T., Sounas, D. L. \& Caloz, C. Artificial Faraday rotation using a ring metamaterial structure without static magnetic field. Appl. Phys. Lett. 99, 03114 (2011).

10. Wang, Z. et al. Gyrotropic response in the absence of a bias field. Proc. Natl Acad. Sci. USA 109, 13194-13197 (2012).

11. Soljačić, M., Luo, C., Joannopoulos, J. D. \& Fan, S. Nonlinear photonic crystal microdevices for optical integration. Opt. Lett. 28, 637-639 (2003).

12. Shadrivov, I. V., Fedotov, V. A., Powell, D. A., Kivshar, Y. S. \& Zheludev, N. I. Electromagnetic wave analogue of an electronic diode. New J. Phys. 13, 033025 (2011)

13. Fan, L. et al. An all-silicon passive optical diode. Science 335, 447-450 (2012).

14. Peng, P. et al. Parity-time-symmetric whispering-gallery microcavities. Nature Phys. 10, 394-398 (2014)

15. Yu, Z. \& Fan, S. Complete optical isolation created by indirect interband photonic transitions. Nature Photon. 3, 91-94 (2009).

16. Lira, H., Yu, Z., Fan, S. \& Lipson, M. Electrically driven nonreciprocity induced by interband photonic transition on a silicon chip. Phys. Rev. Lett. 109, 033901 (2012).

17. Huang, X. \& Fan, S. Complete all-optical silica fiber isolator via stimulated Brillouin scattering. J. Lightwave Technol. 29, 2267-2274 (2011).

18. Kang, M. S., Butsch, A. \& Russell, P. St. J. Reconfigurable light-driven opto-acoustic isolators in photonic crystal fibre. Nature Photon. 5, 549-553 (2011).

19. Fang, K., Yu, Z. \& Fan, S. Photonic Aharonov-Bohm effect based on dynamic modulation. Phys. Rev. Lett. 108, 153901 (2012).

20. Hafezi, M. \& Rabl, P. Optomechanically induced non-reciprocity in microring resonators. Opt. Express 20, 7672-7684 (2012).

21. Poulton, C. G. et al. Design for broadband on-chip isolator using stimulated Brillouin scattering in dispersion-engineered chalcogenide waveguides. Opt. Express 20, 21235-21246 (2012)

22. Wang, D-W. et al. Optical diode made from a moving photonic crystal. Phys. Rev. Lett. 110, 093901 (2013).

23. Kamal, A., Clarke, J. \& Devoret, M. H. Noiseless non-reciprocity in a parametric active device. Nature Phys. 7, 311-315 (2011).

24. Xu, Q., Schmidt, B., Pradhan, S. \& Lipson, M. Micrometer-scale silicon electro-optic modulator. Nature 435, 325-327 (2005).

25. Xu, Q., Manipatruni, S., Schmidt, B., Shakya, J. \& Lispon, M. 12.5 Gbits/s carrier-injection-based silicon micro-ring silicon modulators. Opt. Express 15, 430-436 (2007)

26. Shambat, G. et al. Ultra-low power fiber-coupled gallium arsenide photonic crystal cavity electro-optic modulator. Opt. Express 19, 7530-7536 (2011).

27. Hafezi, M., Demler, E., Lukin, M. \& Taylor, J. Robust optical delay lines with topological protection. Nature Phys. 7, 907-912 (2011).

28. Hafezi, M., Mittal, S., Fan, J., Migdall, A. \& Taylor, J. Imaging topological edge states in silicon photonics. Nature Photon. 7, 1001-1005 (2013).

29. Fang, K., Yu, Z. \& Fan, S. Realizing effective magnetic field for photons by controlling the phase of dynamic modulation. Nature Photon. 6 , 782-787 (2012).

30. Haus, H. A. Waves and Fields in Optoelectronics (Prentice Hall, 1984).

\section{Acknowledgements}

The work was supported in part by AFOSR with YIP award No. FA9550-11-1-0009 and DTRA with YIP award No. HDTRA1-12-1-0022.

\section{Author contributions}

N.A.E. performed the experiment. D.L.S. and N.A.E. designed the structure and conducted the numerical calculations and theoretical modelling. J.S. helped in the selection and modelling of the modulation varactors. A.A. directed and supervised the project. All authors have read and commented on the paper.

\section{Additional information}

Supplementary information is available in the online version of the paper. Reprints and permissions information is available online at www.nature.com/reprints.

Correspondence and requests for materials should be addressed to A.A.

\section{Competing financial interests}

The authors declare no competing financial interests. 